\title{
POBLACIÓN, DESARROLLO HUMANO Y PROCESOS DE URBANIZACIÓN EN LOS PAÍSES MEDITERRÁNEOS DEL MAGREB CENTRAL ${ }^{1}$
}

\author{
Ernesto Cutillas Orgilés \\ Universidad de Alicante \\ Departamento de Geografía Humana \\ ernesto.cutillas@ua.es
}

\begin{abstract}
Resumen: Desde finales del siglo XX se advierten en varios países bañados por el Mediterráneo, pero más intensamente los situados al sur, múltiples cambios sociodemográficos vinculados con la aceleración de sus transiciones demográficas; cuyo reparto por países refleja notables diferencias en las estructuras y en las dinámicas de sus poblaciones. Estos cambios, al margen de las diferencias nacionales, se originan en un contexto general en el que los diferentes ritmos en el aumento de los índices de desarrollo y los procesos de urbanización marcan el futuro de estas sociedades.
\end{abstract}

Palabras clave: Mediterráneo, desarrollo, demografía, Magreb Central, urbanización.

\begin{abstract}
Since the late twentieth century can be seen in several countries bordering the Mediterranean, but more intensely in southern countries, multiple socio-demographic changes associated with the acceleration of demographic transitions, whose breakdown by country shows significant differences in the structure and dynamics of their populations. These changes, regardless of national differences, originate in a general context in which the different rhythms in increasing rates of development and urbanization processes shape the future of these societies.
\end{abstract}

Keywords: Mediterranean, development, demography, Central Maghreb, urbanization.

Recibido: 21-06-13. Aceptado: 25-03-14.

1. Realizado en el marco del Proyecto de Investigación "La reagrupación familiar de los inmigrantes africanos y latinoamericanos en la España mediterránea", Ref. CSO2008-01796, financiado por el Ministerio de Ciencia e Innovación. Investigador Principal: Vicente Gozálvez Pérez. 


\section{Introducción. Un área cultural compleja, delimitada y en transformación}

Desde distintas ciencias sociales y humanas, es fácil asumir que las regiones y los países situados en la ribera sur del Mediterráneo conforman lo que en Geografía se conoce como área cultural. En palabras de Pierre George (1970) estos espacios aluden a la existencia de rasgos culturales genéricos y relativamente homogéneos compartidos entre varios países. En concreto se refiere a los intercambios entre superestructuras de la vida económica y social como por ejemplo, las religiones, los mitos, las actividades intelectuales, artísticas o literarias, etc... Como se desprende de la lectura de Fernand Braudel (1949), mediante estos intercambios entre las orillas del mar y atendiendo a los condicionantes ambientales, similitudes culturales y modos de vida y de relación, los paisajes y las gentes del Mediterráneo conforman una de las civilizaciones más antiguas de la humanidad. En esta línea, López Ontiveros (2008: 93) reflexiona acerca de la capacidad que tienen los pueblos ribereños del mediterráneo de gestar una historia propia, acuñar una forma de vida y sedimentar un pozo específico de valores culturales. Tal es así que Yves Lacoste (2010: 100-101) también señala que, al margen de las diferencias y similitudes, la identidad del Mediterráneo se halla delimitada por el mar, el sol, las fuentes, la maquia, los olivares y las huertas. Esta idílica visión, indiscutible desde el punto de vista geográfico y de los ecosistemas presentes en el territorio, no oculta una compleja realidad sociopolítica y económica vinculada a la falta de seguridad humana que, en mayor o menor grado, afecta a los países del Norte de África y Oriente Medio (Al-Sayyid, 2009: 21). Esta realidad se ve reforzada si, además, se agregan los efectos que las migraciones, la globalización y los imparables procesos de urbanización tienen en las actuales transformaciones sociales a escala global y, en el mundo sur mediterráneo a escala más local. "La división del Islam en grandes áreas culturales [...] tiene cada vez menos sentido. La urbanización y las migraciones mezclaron y estremecieron a las poblaciones tradicionales" (Roy, 2009: 58).

\subsection{Riesgos para la seguridad de las personas y el significado de la evolución demográfica}

El Informe sobre Desarrollo Humano Árabe (2009) señala cuáles son las amenazas que más afectan a los habitantes de estos países a corto y medio plazo ${ }^{2}$. En primer lugar se identifican los riesgos ambientales vinculados al déficit hídrico y a la pérdida de

2. En este trabajo se han agrupado las amenazas en tres grandes grupos. Sin embargo, en el Informe sobre el Desarrollo Humano Árabe (2009) se señalan siete amenazas: 1. Las presiones sobre los recursos ambientales, 2. El papel del Estado en la salvaguarda o vulneración de la seguridad humana, 3. La inseguridad de la población más vulnerable, 4. La vulnerabilidad económica, pobreza y desempleo, 5. Seguridad alimentaria y nutrición, 6. Salud y seguridad humana, 7. La inseguridad e intervención militar extranjera. 
suelos aptos para el cultivo. En segundo lugar se incide en la violencia, ya sea por la ocupación militar, por los conflictos internos o por las medidas represivas de los gobiernos sobre su propia población, o bien contra las poblaciones vecinas. Por último, la tercera dimensión de la seguridad humana hace referencia a las dificultades de la población en el acceso a un empleo digno, a ingresos, a una seguridad social y a una correcta asistencia sanitaria. Frente a este panorama en el que no deben olvidarse los importantes contrastes sociopolíticos y culturales, hay que añadir la línea de fractura económica que separa la Europa desarrollada del mundo árabe más pobre y cuyo Producto Interior Bruto es once veces inferior al de la Unión Europea (Mutin, 2009: 14); o la notable influencia de los estados petroleros del Golfo Pérsico, ya sea mediante la atracción de inmigrantes o a través de sus inversiones directas, que en el año 2009 ya estaban muy próximas a las que se realizan desde la Unión Europea (Semmoud, 2010: 201). Esta descripción se completa bajo la persistencia de infundados posicionamientos que advierten, no sólo para los países occidentales si no también para los países árabes, sobre las nefastas consecuencias de tipo maltusiano que tiene el crecimiento demográfico de la población en cuanto que supone potenciales choques étnico/religiosos (Huntington, 1997) o conflictos en el acceso y consumo de recursos naturales, particularmente de agua o hidrocarburos (Mutin, 2009), (Bennafla; Pàges-el Karoui, Sanmartin: 2011). Sin embargo, y atendiendo a las actuales estructuras y proyecciones de la población, la "bomba demográfica" que supone el mundo árabe está desactivada desde hace décadas, si es que alguna vez estuvo activada. Ello es debido a la importante reducción de su fecundidad que ya se advertía desde la década de 1970 (Lacoste, Lacoste, 1991), y que contribuyó a que en la actualidad los índices de fecundidad generales no superen en más de un punto los que se registran al norte del Mediterráneo "... lo que viene a corroborar la bipótesis del final de la explosión demográfica del mundo árabe y del sur del Mediterráneo" (Courbage, 2009: 74).

Argüir a la evolución demográfica un riesgo político y/o ambiental que no es real no hace sino confirmar la existencia de posturas ideológicas estigmatizantes e interesadas que se nutren en planteamientos ambientalistas para no cuestionarse decididamente la sostenibilidad y viabilidad del actual sistema-mundo y las relaciones de poder. Así, descartando de plano el factor desestabilizador del crecimiento demográfico en los países del sur del Mediterráneo, cabe indagar sobre los efectos que en la estructura demográfica de la población tienen la evolución de las transiciones demográficas, los distintos grados de desarrollo, los modos de vida y los procesos de urbanización, fenómenos todos ellos que inciden en la necesidad de cambios sociopolíticos y de seguridad en la región «las revueltas populares en algunos países árabes como Túnez, Egipto, Libia o Siria, [...] tampoco resultan ajenas en su origen al creciente malestar que supone una brecha social cada vez más visible, acentuada por una presión demográfica también en aumento y que encuentra su mejor exponente en los suburbios de las grandes ciudades" (Méndez, 2011: 33-34). No en vano, las ciudades, caracterizadas por unas infraestructuras insuficientes y obsoletas, con barrios de gran hacinamiento, insalubres e insegu- 
ros (Informe sobre el Desarrollo Humano Árabe, 2009: 35-34) son los lugares a tener en cuenta en relación a los procesos de cambio y malestar social que se observa en los países del sur y este del Mediterráneo, especialmente cuando la población joven-adulta es el grupo de mayor crecimiento de las poblaciones árabes.

\subsection{Características de las dinámicas demográficas recientes}

Desde comienzos de la década de $1950^{3}$, la proporción de habitantes en los países bañados por el Mediterráneo ha registrado un descenso sostenido en el tiempo respecto del total de la población mundial ${ }^{4}$. En ese año sus 206 millones de habitantes representaban el $8,15 \%$ de la población mundial. Transcurridas seis décadas y en un entorno internacional caracterizado por el mayor crecimiento demográfico de la humanidad, los habitantes de los países mediterráneos alcanzan los 470 millones, pero sólo representan el $6,82 \%$ de todos los habitantes del planeta. En efecto, en poco menos de seis décadas la población se ha doblado, pero esa tendencia refleja un comportamiento demográfico bastante desigual con respecto a otras regiones mundiales que han experimentado mayores crecimientos relativos, como Asia Meridional o África Subsahariana, pero especialmente esta tendencia manifiesta una desigualdad interior que se refleja en tamaños poblacionales muy dispares (como por ejemplo los más de 81 millones de habitantes de Egipto o los poco más de 4 millones en el Líbano), en la coexistencia de varias velocidades en las transiciones demográficas claramente identificables entre la ribera sur y norte del Mediterráneo y en unas complejas estructuras poblacionales que sin duda van a condicionar más que nunca los próximos lustros de estos países y que, siguiendo a Domingo (2011: 102), suponen un desplazamiento de lo económico a lo demográfico como principal factor que condicionará su futuro. No en vano, los retos que tienen ante sí los países del mundo musulmán y que quedaron plasmados en el Informe sobre Desarrollo Humano Árabe (2009) tienen una componente demográfica de primer nivel.

Tomando como referencia los países norteafricanos más poblados, y al margen de la opinión pública mayoritaria, cabe señalar que en la década 2000-2010 registraron incrementos de población relativamente moderados en un contexto mundial de gran crecimiento económico, además de presentar índices de fecundidad bajos que superan por

3. Es conveniente señalar que el grado de fiabilidad de la información estadística proporcionada por instituciones oficiales siempre debe ser cuestionado por razones vinculadas a la naturaleza de la fuente, metodología de obtención de la información, grado de desagregación, periodicidad, entre otros. No obstante, para esta parte del trabajo se ha optando por utilizar y aceptar como más fiables los datos que se recogen en los Informes sobre las Perspectivas Demográficas Mundiales (World Population Prospects) elaborados por el Departamento de Economía y Asuntos Sociales de las Naciones Unidas del año 2011 y los que figuran en los Informes de Desarrollo Humano que se elaboran dentro para el Programa de las Naciones Unidas para el Desarrollo (PNUD).

4. Egipto, Turquía, Francia, Italia, España, Argelia, Marruecos, Siria, Grecia, Túnez, Israel, Libia, Croacia, Líbano, Palestina, Bosnia-Herzegovina, Albania, Eslovenia, Chipre, Montenegro y Malta. 
pocas décimas el umbral de reemplazo generacional5: Marruecos y Argelia, 2,38 hijos por mujer o Túnez 2,04; índices que por ejemplo son inferiores a los que en España se registraban a comienzos de los años $1980^{6}$ (tabla 1). Centrándonos en estos países magrebíes, el descenso de fecundidad en la última década mantiene dos contrastes muy notables. En primer lugar, y en paralelo a esa reducción generalizada, se ha producido en un contexto regional en el que los países de la ribera norte del Mediterráneo registraban significativos repuntes de su fecundidad como consecuencia de los notables saldos migratorios acumulados, especialmente en España (2.250.000 personas) e Italia (1.999.000 personas), y se ha producido también durante el importante período de crecimiento económico que se registró en estos países en los primeros años de la década. En segundo lugar, esa reducción de la fecundidad se debe circunscribir a un espacio norteafricano que, salvo en Marruecos, en su conjunto muestra una ligera desaceleración de los saldos migratorios negativos, más acentuada en países como Túnez o Egipto (tabla 1).

Por otro lado, la expectativa de vida al nacer, como fenómeno demográfico clave en la dinámica y en el desarrollo de las poblaciones, también refleja significativas tendencias de cambio. Cambio hacia el envejecimiento de las poblaciones que se traduce con unos incrementos medios en la esperanza de vida bastante superiores en los países de mayoría musulmana que los que se observan para el mismo periodo en los países europeos. Estos últimos, sin embargo, parten de una situación más ventajosa puesto que al final del periodo estudiado todos ellos superan holgadamente la expectativa de vida al nacer de ochenta años, mientras que para la práctica totalidad de países de Oriente Próximo y el Magreb esa cifra todavía queda lejos de ser alcanzada pese a que ninguno de ellos registra ya esperanzas de vida inferiores a los setenta años; como se demuestra al observar que los países que más baja la tenían al comienzo del siglo XXI, son aquellos que más la han aumentado: Marruecos, Egipto y Turquía (tabla 1). Así, de mantenerse las mismas condiciones (sociales, económicas y/o ambientales) que propician dicho incremento, sería posible una convergencia hacia la equiparación en la esperanza de vida de los habitantes del espacio mediterráneo. Si bien la persistencia de conflictos armados y/o latentes, las políticas represivas interiores o exteriores o la permanencia de importantes desequilibrios sociales, no hacen sino incrementar la inseguridad de la población y retrasar la paulatina equiparación de las expectativas de vida entre ambas orillas del Mediterráneo.

5. Se estima que 2,13 hijos por mujer en edad de tener descendencia es la cifra necesaria para garantizar el reemplazo generacional en una sociedad donde el saldo migratorio es nulo.

6. Según el departamento de Economía y Asuntos sociales de las Naciones Unidas, para el periodo 19751980 el número medio de hijos por mujer en España era de 2,55 hijos. 


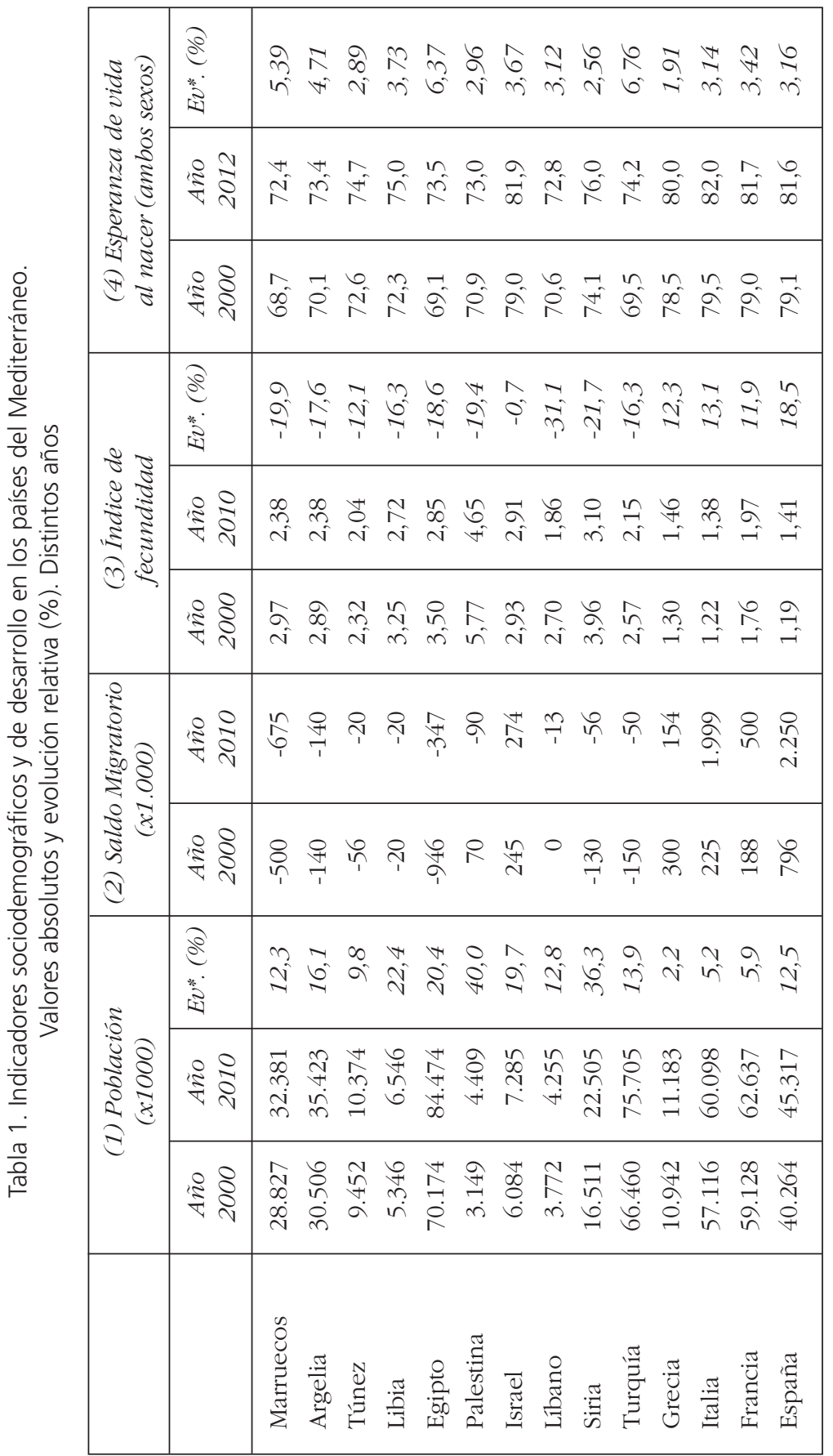






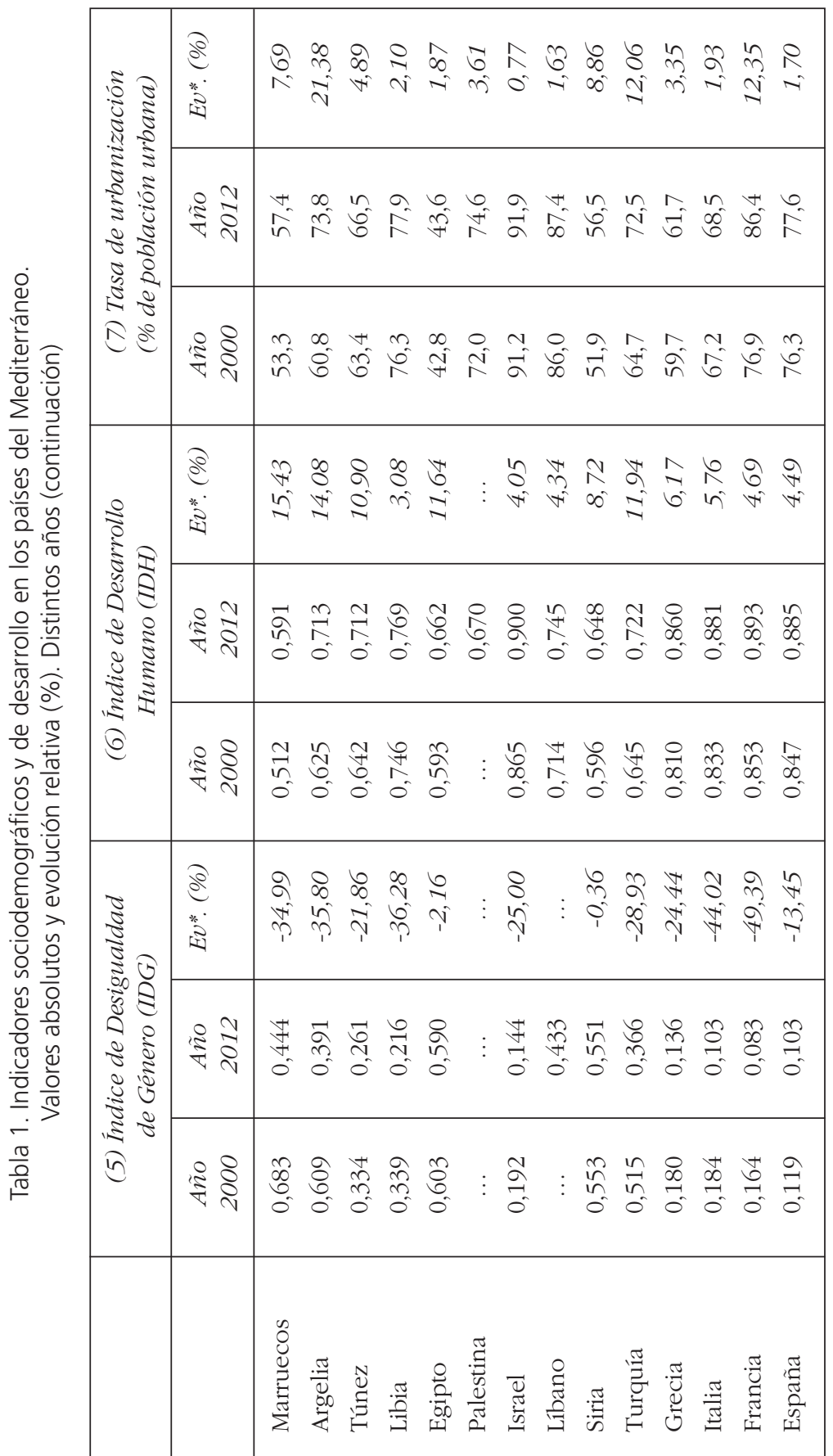






\subsection{Las estructuras demográficas como exponente de las transformaciones sociales}

La dinámica demográfica actual de los países del Mediterráneo, en la que influyen las diferencias relativas a la fecundidad, a las migraciones y al envejecimiento, se refleja nítidamente en el reparto de las notables asimetrías en las estructuras demográficas de las poblaciones (mapa 1); a las cuales se ha llegado tras unas rápidas transiciones demográficas en los países musulmanes, y muy especialmente en los del Magreb Central o Histórico, y cuyos efectos a corto plazo ya se manifiestan sin necesidad de esperar una nueva generación debido a que los dividendos demográficos ${ }^{7}$ se consolidan con estructuras demográficas cada vez más adultas y alejan a estos países de otras décadas del siglo pasado en las que su composición poblacional se nutría fundamentalmente de niños y jóvenes, tal y como en la actualidad sucede en otros países musulmanes ${ }^{8}$.

No en vano, y atendiendo a la estructura por edades de la población se pueden observar las importantes diferencias que mantiene la población mediterránea por grandes áreas geográficas, destacando por su complejidad el Mediterráneo Oriental. A ese respecto, se pueden observar países como Siria o Palestina cuya población joven, de 0 a 19 años de edad, el 47,9\% y el 54,6\% respectivamente, es prácticamente similar o incluso superior a su población adulta, hecho que se produce precisamente en los países que registran los menores índices de desarrollo humano de la región, y cuyos conflictos, interiores y exteriores, no hacen sino comprometer aún más la seguridad de la población joven, que precisamente es la más vulnerable. Por ese motivo, y desde un punto de vista estrictamente sociodemográfico cabe plantearse qué perspectivas de futuro hay para la población de unos países que registran unas edades medias de 21,1 años y de 18,1 años y para los que todavía no se han hecho efectivas las medidas necesarias para garantizar los derechos 9 más básicos de su población. Ampliando la escala de análisis al conjunto del Mediterráneo, estos contrastes demográficos en cuya naturaleza se hallan condicionantes políticos, culturales, históricos, económicos o territoriales, son los mismos que, en menor o mayor grado, explican las notables diferencias actuales entre los países situados en la ribera sur del Mediterráneo y los del norte.

Así, frente a unos países europeos cuyos promedios de población joven apenas logran alcanzar el 20\% de su población (excepto en Francia) y sus edades medias se sitúan en torno a los 40 años de edad, se encuentran el resto de países en los que en ningún caso, salvo

7. El dividendo o bono demográfico hace referencia a la situación demográfica en la cual la población potencialmente activa es superior a la población dependiente, niños y ancianos.

8. De hecho, según las perspectivas de la población mundial ofrecidas por las Naciones Unidas para el año 2010, el 57,55\% de la población de Afganistán tiene menos de 19 años, las mismas edades que el 50,60\% de la población de Sudán o el 46,57\% de la de Pakistán. Estos datos, junto con unas edades medias respectivas de 16,6 años, 19,7 años y 21,7 años, corroboran el importante peso de la población infantil en estos países.

9. Declaración Universal de los Derechos Humanos, 1948. http://www.un.org/es/documents/udhr/ 
en Israel, se llega a una edad media superior a los 30 años y cuya población joven se sitúa siempre por encima del 30\% del total de sus habitantes (mapa 1). La clave a corto y medio plazo ${ }^{10}$ de estas brechas en la distribución de las estructuras demográficas por edades se centra en la relación (favorable o no) de la población potencialmente activa: adultos, con la población potencialmente inactiva: mayores y jóvenes (Domingo, Gil, Bayona, 2011). Tal es así que una composición por edades como la que se describe para los países europeos conduce al envejecimiento demográfico y por lo tanto a la inactividad, mientras que las composiciones propias de los países norteafricanos y del Mediterráneo oriental presentan dividendos demográficos muy favorables a la actividad en un contexto de aumento de la esperanza media de vida y de significativa reducción de la fecundidad, especialmente en unos países cuya población femenina en el medio urbano registra índices de fecundidad similares a los europeos, y que comienzan a repuntar tímidamente (Ouadah-Bedidi, Vallin, Bouchoucha, 2012). Esta situación se complementa con el efecto que las migraciones internacionales introducen en un territorio en donde en una parte se tiende al envejecimiento (Europa) mientras que en otra (Norte de África y Oriente Próximo), y como consecuencia de la reducción de su fecundidad, los grupos de edad adulta son los que presentan las mayores perspectivas de crecimiento, y en ese sentido cabe advertir que los estados no han sido capaces de hacer frente a las necesidades de la población, fundamentalmente las laborales, lo que ha contribuido a profundizar más en los conflictos latentes y a favorecer las revueltas populares (Ossorio, 2011: 66-67).

A ese respecto, ¿es posible que las migraciones internacionales corrijan esos desequilibrios? En efecto, no parece factible que las migraciones de reemplazo sean las que solucionen los retos demográficos para los países que envejecen rápidamente (Pool, 2007: 34), ni que en el sur sean aquéllas que faciliten la contención social en poblaciones y territorios profundamente desiguales (Domingo, 2011). Más bien cabe indagar acerca de las posibilidades reales que existen en los países del norte de implementar políticas eficaces de conciliación laboral y familiar, mientras que en los países del sur y el este del Mediterráneo profundizar en las reivindicaciones compartidas por la sociedad civil tales como el desmantelamiento de los estados autoritarios, la lucha contra la corrupción, la derogación de las leyes de emergencia, el fin de los sistemas monopartidistas, etc... (Ossorio, 2011: 67). Sin olvidarse, desde una perspectiva geodemográfica, de compatibilizar por un lado la necesidad de inserción laboral de una generación de población joven con niveles formativos superiores a los de décadas anteriores y; por otro lado, mejorar la situación de las mujeres, quienes cada vez asumen mayor protagonismo en las economías familiares, especialmente en las ciudades (Courbage, 2009), (Locoh; Ouadah, 2010) lo que sin duda es decisivo en la actual estructura demográfica y en la futura.

10. Las proyecciones de población muestran cómo sería el futuro demográfico de un país de mantenerse sin cambios los movimientos naturales y espaciales de la población. Las proyecciones corto plazo (hasta 10 años) son más fiables y muestran las consecuencias más inmediatas de la evolución de los fenómenos demográficos. A medio plazo (a partir de 10 años) se puede observar el resultado demográfico de los actuales comportamientos respecto de la fecundidad y mortalidad y de la estructura de la población. 




Mapa 1. Distribución de la edad media y de la estructura demográfica por edades de la población en los países mediterráneos. Valores absolutos y relativos (\%), año 2010.

Fuente: DGA. Naciones Unidas (2011). World Population Prospects: 2010. Elaboración propia.

\subsection{Diferencias en el reparto por sexos de la población: hacia una lenta feminización de la sociedad}

Tomando como referencia el conjunto de la población del Magreb Histórico, aparentemente más estable, se evidencia que su estructura por sexo y edad presenta un equilibrio, en parte similar al que se registra en los países europeos del Mediterráneo Occidental (gráfico 1).

Obviando las diferencias etarias en la distribución geográfica de la población, el reparto por sexos muestra que en 2010 las mujeres representan el 50,2\% de la población en el Magreb Central, mientras que en el conjunto de Francia, Italia y España (países europeos del Mediterráneo occidental) la cifra alcanza el 51,0\%. Las proyecciones de las Naciones Unidas a corto plazo muestran que para 2020 esas cifras relativas, a pesar del incremento absoluto de población, serán bastante similares: el 51,1\% y el 50,7\%, respectivamente. Lo interesante de estos datos es que confirman el mantenimiento del equilibrio por sexos, especialmente en las edades potencialmente activas y, por lo tanto, 


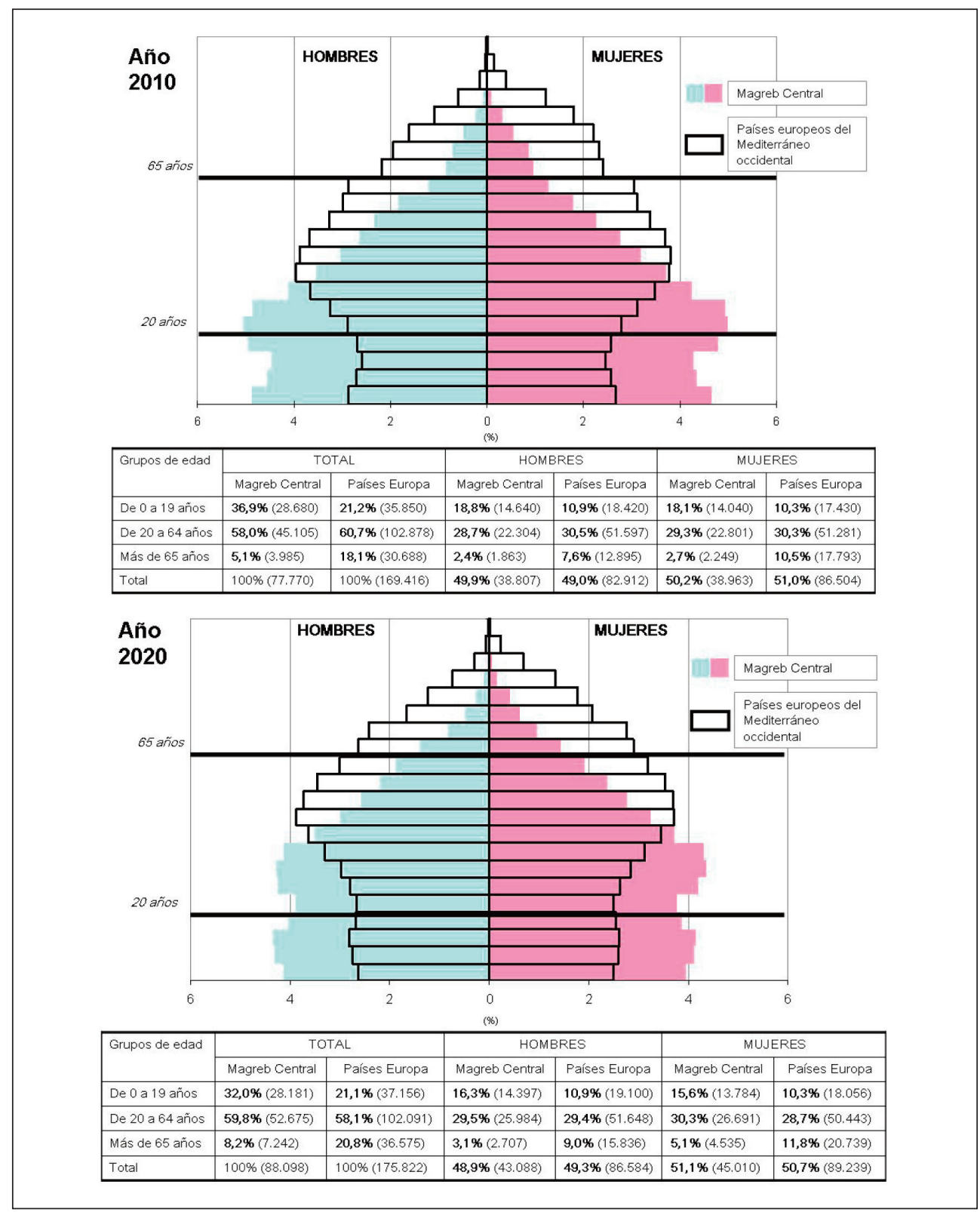

Gráfico 1. Estructura por edad y sexo de los países del Magreb Central (Argelia Marruecos y Túnez) y los países europeos del Mediterráneo Occidental (Francia, Italia y España), años 2010 y 2020.

Fuente: DGA. Naciones Unidas (2011). World Population Prospects: 2010. Elaboración propia. 
en aquellas en las que las mujeres cada vez participan más de la actividad. De hecho, y a diferencia de Europa, para el año 2020 habrá una mayor proporción de mujeres magrebíes con edades comprendidas entre los 20 y los 64 años de edad: el 30,3\% que hombres magrebíes: 29,5\%, y mujeres y hombres europeos: $28,7 \%$ y 29,4\%, respectivamente. Esta feminización de la población a edades adultas en el conjunto del Magreb Central, que también se observa en otros países musulmanes, representa un cambio cultural de primer nivel y puede llegar a repercutir decisivamente en los roles y en la conformación de nuevos hogares debido principalmente a un aumento de la escolaridad de las niñas ${ }^{11}$ y por las modificaciones en los patrones de la sexualidad o la nupcialidad, como por ejemplo el aumento de mujeres que utilizan métodos anticonceptivos, el aumento de la edad del matrimonio o la probabilidad de que cada vez hayan más mujeres que opten por no casarse; ya que en las decisiones que motivan estos comportamientos se observa que cada vez más prevalecen las elecciones individuales frente a presiones culturales o económicas (Courbage; Todd: 2009), (Locoh; Ouadah: 2010).

\subsection{Implicaciones socioterritoriales del desarrollo humano en la región}

Pese a las limitaciones que pudiera ofrecer el Índice de Desarrollo Humano (IDH) ${ }^{12}$ para medir la calidad de vida de las personas, especialmente en los países mediterráneos (Reig-Martinez, 2013), es cierto que su evolución reciente y reparto en los países de la cuenca del Mediterráneo ofrece unas tendencias muy significativas que se vinculan con indicadores sociodemográficos básicos referentes a la estructura y a la dinámica de las poblaciones "el IDH ha demostrado ser un instrumento flexible capaz de medir diferencias entre países en su nivel de desarrollo bumano y cambios en el desarrollo bumano a lo largo del tiempo" (Griffin, 2001: 33). Tanto las cifras absolutas que alcanza dicho indicador durante la primera década del siglo XXI, así como su evolución relativa permiten identificar contrastes muy significativos entre los países de la región. Marruecos, Argelia y Túnez, pese a partir con cifras bajas en el IDH, son los países que han registrado los mayores incrementos relativos, especialmente en el caso de Argelia: 14,08\%, y de Marruecos: 15,43\%; y según los datos para el año 2012 aportados por el Programa de las Naciones Unidas para el Desarrollo, sigue siendo este último el país con menor IDH de la región (0’591), hecho que continua dando vigencia

11. Según el informe sobre el cumplimiento de los Objetivos del Milenio en el norte de África para el año 2011, se ha alcanzado ya o está en vías de ser alcanzado para el 2015 la enseñanza primaria universal y la equidad en la matriculación de niñas en la escuela primaria. http://mdgs.un.org/unsd/mdg/Resources/Static/Products/Progress2011/11-31333\%20\%28S\%29\%20MDG\%20Report\%202011_Progress\%20Chart\%20LR.pdf

12. Según el Programa de las Naciones Unidas para el Desarrollo (PNUD) el Índice de Desarrollo Humano (IDH) es un indicador compuesto que combina los valores vinculados a la esperanza de vida al nacer, años promedio de instrucción, años de instrucción esperados e ingresos brutos per cápita, con ellos se obtiene información referente a la salud, la educación y el estándar de vida. 
a la consideración del estrecho de Gibraltar como el "Río Grande" de Europa que separa dos realidades económicas y sociales contrapuestas (Mansvelt-Beck; de Mas, 2001). Por otro lado, Túnez y Argelia son los países africanos que también destacan en la evolución relativa de este indicador puesto que alcanzan valores absolutos propios de países de Desarrollo Humano Medio, y los sitúa por encima del desarrollo humano que se registra en un país emergente como por ejemplo China ${ }^{13}$. Por su parte, en los países de la ribera oriental se observa, con la excepción de Siria, que el IDH alcanza cifras de países de desarrollo medio en Egipto $(0,662)$ o Palestina $(0,670)$, de desarrollo alto como en el Líbano $(0,745)$ o de desarrollo muy alto en Israel $(0,900)$ que lo sitúa a niveles superiores a los de Europa occidental.

Sin embargo, y llegados a este punto, resulta clarificador comprobar cómo los países que más han aumentado su IDH durante el periodo estudiado, resultan ser aquellos que más han reducido sus índices de fecundidad (tabla 1). Esta situación permite observar una triple tendencia que clarifica la relación que mantiene el desarrollo humano con la fecundidad en los países mediterráneos y facilita una mejor descripción del rol femenino en estas sociedades por cuanto que permite intuir importantes cambios culturales y mayor diversidad en los modos de vida. Así, un primer grupo de países constituido por los que componen el Magreb Histórico: Argelia, Marruecos y Túnez se caracterizan por las mayores reducciones en la fecundidad al tiempo que son los que más han aumentado su IDH, dicha tendencia, aunque algo más atenuada, también se observa en dos gigantes demográficos del Mediterráneo, Egipto y Turquía, precisamente los referentes geopolíticos de la región. Un segundo grupo de países conformado por Líbano, Siria y Libia comparten la importante reducción de sus índices de fecundidad pero en un contexto de escaso crecimiento de su IDH. En tercer lugar los países europeos del mediterráneo occidental aumentan sustancialmente sus índices de fecundidad mientras que sus niveles de desarrollo humano apenas registran variaciones significativas. Este panorama adquiere mayor dimensión en el Magreb Histórico al constatar que Marruecos y Argelia son los países norteafricanos que con mayor intensidad han reducido su Índice de Desigualdad de Género (IDG) ${ }^{14}$ aunque todavía éste no se ha situado a los niveles registrados para Túnez, y en menor medida a los de Libia incluso tras la guerra, precisamente los dos estados menos desiguales de los países de mayoría musulmana de la ribera meridional y oriental del Mediterráneo.

13. Para el año 2012 China tiene un IDH de $0,699$.

14. Según el Programa de las Naciones Unidas para el Desarrollo (PNUD) el Índice de Desigualdad de Género (IDG) es un indicador compuesto que pone de manifiesto las diferencias existentes en la distribución de los logros alcanzados por mujeres y hombres. Para ello se emplean cinco indicadores: mortalidad materna, fecundidad adolescente, escaños en el parlamento, nivel de instrucción (secundario y superior) y participación en la fuerza laboral que muestran tres dimensiones claves en el bienestar femenino: salud reproductiva, empoderamiento y mercado laboral. 


\section{Litoralización demográfica y los contrastes en la densidad de la población del Magreb Central}

La tendencia que vincula la fecundidad, el desarrollo humano y una paulatina, aunque aparentemente imparable, tendencia a la equiparación de género se puede constatar con mayor facilidad en las áreas urbanas de la región puesto que son los espacios urbanos en donde tradicionalmente se han iniciado los procesos de cambio social y/o cultural, y desde allí se han generalizado y difundido. Dicha situación contrasta con un medio rural en donde persisten notables diferencias respecto a las tasas de alfabetización y acceso al agua potable o a la electricidad (Informe Desarrollo Humano Árabe, 2009).

Los países del Magreb Central, al igual que el resto de los países norteafricanos, presentan contrastes extremos en el reparto interno de su población, siendo ésta una de las regiones del mundo que mayores diferencias presenta en la distribución de su densidad demográfica. Los desequilibrios están motivados principalmente por razones climáticas, pero por necesidades e intereses políticos-económicos pueden aparecer emplazamientos habitados en zonas desérticas o semidesérticas como en la capital de la wilaya (o provincia) argelina de Béchar por la conexión ferroviaria con Orán, en los campamentos de refugiados saharauis en Tindouf, la presencia de oasis dedicados a las actividades primarias o la existencia de áreas industriales dedicadas a la extracción de recursos naturales como en zonas de las gobernaciones tunecinas de Kasserine para hidrocarburos o la marroquí de Sus-Masa-Dra para minerales metálicos. En cualquier caso, las densidades en la región son muy extremas, así por ejemplo se pueden encontrar wilayas argelinas como por ejemplo Tamanrasset con una superficie de 556.185 $\mathrm{km}^{2}$ (superior a la de España) y cuya densidad es de 0,32 habitantes por kilómetro cuadrado, o como Illici con $285.000 \mathrm{~km}^{2}$ (más de tres veces la superficie de Andalucía) y una densidad tan sólo de 0,18 habitantes por kilómetro cuadrado; al otro extremo estarían las elevadas densidades de las principales áreas urbanas como los 2.308,87 habitantes por kilómetro cuadrado de la región de Gran Casablanca, los 2.511,05 de la wilaya de Argel o, sobre todo, los 3.434,03 habitantes por kilómetro cuadrado de la gobernación de Túnez (mapa 2). Estas enormes diferencias en la ocupación del territorio son un claro ejemplo de las asimetrías que se pueden encontrar a una escala más local; si bien a ese nivel las disparidades están relacionadas con la gobernanza y la ordenación del territorio a nivel nacional, regional y local.

En efecto, la primacía de las grandes aglomeraciones condiciona la organización territorial de los países y se manifiesta a través de un importante proceso de metropolitanización (Semmoud: 2010: 231) que agrava los antiguos desequilibrios socioterritoriales relacionados con los grandes vacíos demográficos y crea, a la vez, otros nuevos desequilibrios vinculados al bienestar social de la población urbana y a la gestión de los procesos migratorios internos de los países que se encuentran en una transición urbana avanzada, como es el caso de Argelia y de Marruecos, o bien que han comple- 
tado definitivamente la transición campo-ciudad como en Túnez (Troin et al., 2006: 75). Siguiendo a Dumont y Montenay (2011: 43), se puede afirmar que en los últimos años las desigualdades en el poblamiento de los países del Magreb Central, lejos de mantenerse, se han fortalecido en la medida que los procesos de urbanización aumentan el peso relativo de las regiones que tradicionalmente ya estaban muy pobladas.

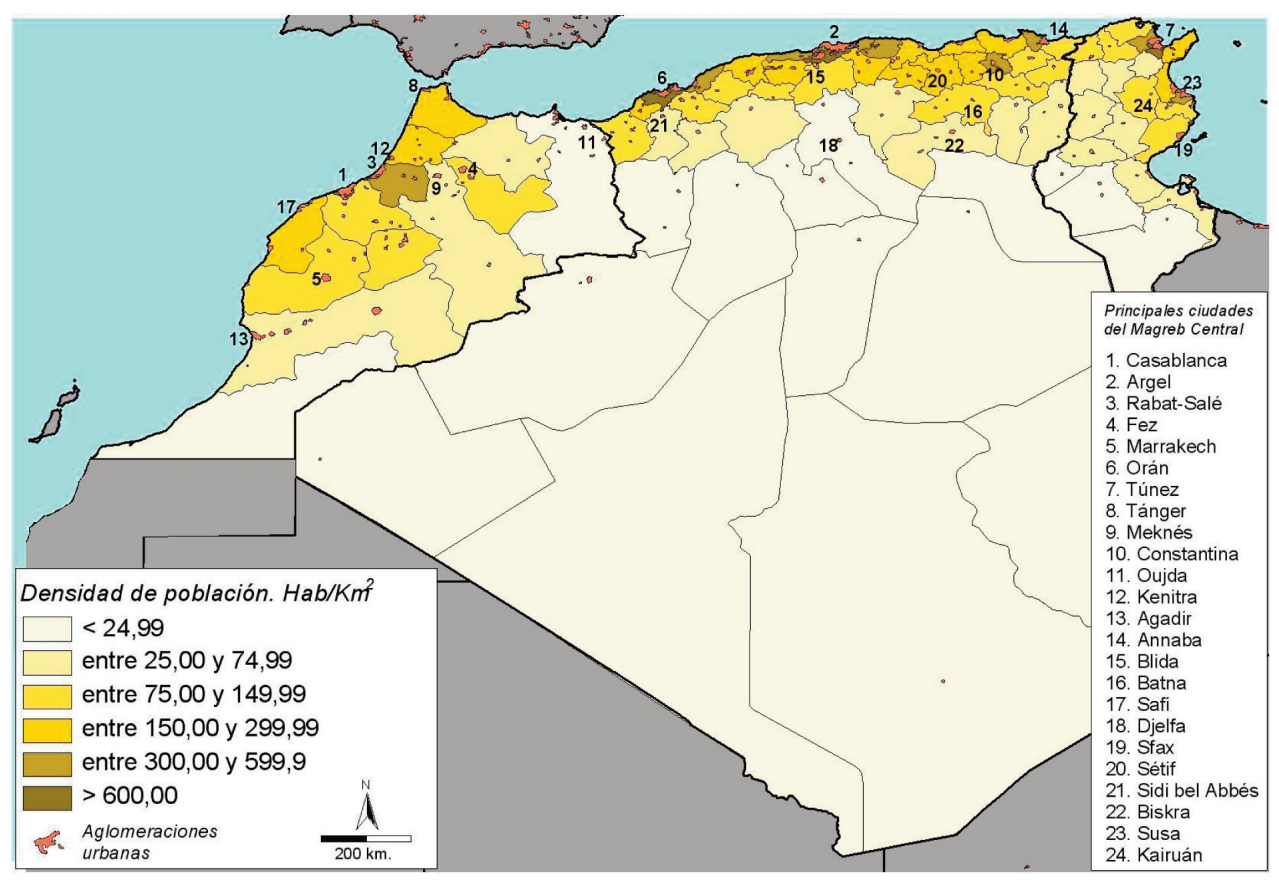

Mapa 2. Principales ciudades y distribución regional de la densidad de población en el Magreb Central (habitantes/kilómetro cuadrado).

Fuente: Argelia: ONS, Office National des Statistiques: statistiques sociales, Recensement Général de la Population, 2008 http://www.ons.dz/. Marruecos: Haut-Commissariat au Plan, Recensement Général de la Population et de l'Habitat, 2004 http://www.hcp.ma/. Túnez: Institut National de la Statistique, Recensement de la Population, 2004. http://www.ins.nat.tn/. Elaboración propia.

Así, la litoralización de las poblaciones, es el elemento común que caracteriza a los sistemas de poblamiento de los países del Magreb histórico. En mayor o menor grado todos los países mantienen una estrecha relación con sus fachadas marítimas, lo que explica las elevadas densidades demográficas que se registran en sus regiones litorales y pre-litorales y que se remontan a la época de la colonización cuando las potencias occidentales ejercían su dominio desde las principales ciudades hacia el interior para dar paso, tras las independencias, a una mayor litoralización económica basada en el re- 
descubrimiento de sus recursos naturales, el crecimiento de las actividades industriales del litoral, un mayor posicionamiento internacional de los puertos de mercancías y la pujanza de algunos complejos turísticos de balneario (Semmoud: 2010: 241). Como contraste a ese dinamismo, los principales problemas urbanos se manifiestan con los procesos territoriales y funcionales asociados a la importante urbanización de las áreas periféricas de las grandes ciudades; mientras que en las medinas la tendencia a su vaciamiento, degradación y la aparición de redes de solidaridad islámica que asumen el rol asistencial de los Estados, refuerza la idea de una importante dualidad morfológica y social en las ciudades árabes (Bennafla, Pagès-El Karoui; Sanmartin, 2011: 73-76).

\subsection{El peso funcional de las grandes aglomeraciones urbanas y sus tendencias en el Magreb Central}

Según las perspectivas de la urbanización mundial de las Naciones Unidas, hay en el Magreb Central nueve aglomeraciones urbanas que superan cada una los 750.000 habitantes ${ }^{15}$. Desde inicios de la década del dos mil, los procesos de urbanización en los países del Mediterráneo han avanzando de una manera muy dispar y con contrastes territoriales muy notables que permiten augurar una década de paulatino descenso en el ritmo de crecimiento urbano de algunos países del sur y del este del Mediterráneo que ya registraron su "explosión urbana" y que cuentan con tasas de población urbana similares o superiores a la de los países europeos como sucede en Libia: el 77,9\% o el Líbano: el 87,4\%; mientras que en otros países todavía tienen bastante margen para aumentar dicha tasa, especialmente los de mayor dimensión y/o los que aún presentan los índices más bajos de la región como es el caso de Egipto: el 43,6\% o de Siria: el 56,5\%.

En los últimos años Argelia ha sido el país que ha registrado el mayor crecimiento de población urbana de la región, un 21,38\%; lo que ha deparado que éste sea el país más urbanizado del Magreb histórico con una tasa de población urbana del 73,8\% frente al 66,5\% de Túnez o el 57,4\% de Marruecos (tabla 1). Sin embargo, y pese a que el ritmo de la urbanización no es homogéneo entre estos países, se puede observar, mediante los datos disponibles (tabla 2$)^{16}$, una tendencia que al menos ha sido muy destacada hasta mediados de la primera década de los años dos mil y que muestra que el crecimiento urbano más intenso no se ha producido tanto en las capitales estatales y/o capitales económicas: Rabat 1,46\%, Argel 1,26\%, Túnez, 1,00\%, Casablanca 0,83\% u Orán:

15. Casablanca: 3.009.000, Argel: 2.851.000, Rabat: 1.807.000, Fez: 1.065.000, Marrakech: 919.000, Tánger: 790.000, Agadir: 786.000. Túnez: 777.000, Orán: 776.000.

16. Los datos recogidos en la tabla 2 se han obtenido en los portales estadísticos de los respectivos servicios nacionales de estadística. Conviene advertir la dificultad que entraña conseguir una información relativamente estandarizada, homologable, del mismo período y fiable. Por ese motivo, los datos expresados hay que considerarlos con cierta cautela y asumir que los valores sólo muestran tendencias generales. 
0,83\%; sino más bien los crecimientos interanuales de población urbana se han producido bien en municipios del entorno de estas aglomeraciones, bien en capitales administrativas de otras regiones beneficiadas recientemente por inversiones extranjeras, por la puesta en valor de recursos de la zona y por su situación estratégica o bien por políticas descentralizadoras. Tal es el caso de Djelfa y Blida en Argelia con un crecimiento interanual de la población de 5,30\% y 2,89\%, respectivamente; al sur de Marruecos Agadir con un crecimiento del 3,11\% y Tánger, al norte, con un 3,02\%; o los casos de las ciudades tunecinas de Susa en el litoral: 3,71\% o más hacia el interior Kairuán con un crecimiento interanual de 2,48\%, aunque en ambos casos se trata de ciudades relativamente próximas a su capital nacional.

Tabla 2. Población registrada en las principales ciudades del Magreb Central. Varios años Tasa de crecimiento anual acumulado (\%)

\begin{tabular}{|c|c|c|c|c|c|c|c|}
\hline & \multicolumn{3}{|c|}{ Argelia } & & \multicolumn{3}{|c|}{ Marruecos } \\
\hline & Año 1998 & Año 2008 & $r(\%)$ & & Año 1994 & Año 2004 & $* r(\%)$ \\
\hline Argel & 2.086 .212 & 2.364 .230 & 1,26 & Casablanca & 2.713 .169 & 2.946 .440 & 0,83 \\
\hline Orán & 735.166 & 803.329 & 0,89 & Rabat (Salé) & 1.195 .227 & 1.381 .666 & 1,46 \\
\hline Constantina & 479.122 & 448.028 & $-0,67$ & Fez & 772.184 & 946.815 & 2,06 \\
\hline Annaba & 331.539 & 342.703 & 0,33 & Marrakech & 669.043 & 823.154 & 2,09 \\
\hline Blida & 249.553 & 331.779 & 2,89 & Tánger & 497.147 & 669.685 & 3,02 \\
\hline Batna & 246.800 & 289.504 & 1,61 & Meknés & 393.791 & 469.169 & 1,77 \\
\hline Djelfa & 158.679 & 265.833 & 5,30 & Oujda & 357.278 & 400.738 & 1,15 \\
\hline Sétif & 214.842 & 252.127 & 1,61 & Kenitra & 292.453 & 359.142 & 2,08 \\
\hline Sidi bel Abbès & 183.931 & 210.146 & 1,34 & Agadir & 254.865 & 346.106 & 3,11 \\
\hline Biskra & 177.060 & 204.661 & 1,46 & Safi & 262.276 & 284.750 & 0,83 \\
\hline \multicolumn{5}{|c|}{$\left({ }^{*} \mathrm{r}\right)=$ Tasa de crecimiento anual acumulado: } & \multicolumn{3}{|c|}{ Túnez } \\
\hline \multirow{2}{*}{\multicolumn{4}{|c|}{$r=\sqrt[n]{\frac{P^{t+n}}{P^{t}}}-1$}} & & Año 1984 & Año 2004 & $r(\%)$ \\
\hline & & & & Túnez & 596.654 & 728.453 & 1,00 \\
\hline \multirow{2}{*}{\multicolumn{4}{|c|}{ Donde $\mathrm{n}=$ número de años, }} & Sfax & 231.911 & 265.131 & 0,67 \\
\hline \multirow{2}{*}{\multicolumn{4}{|c|}{$\begin{array}{l}\mathrm{P}^{\mathrm{t}+\mathrm{n}}=\text { la población final del periodo } \\
\text { estudiado, } \mathrm{P}^{\mathrm{t}}=\text { la población inicial } \\
\text { del periodo estudiado }\end{array}$}} & Susa & 83.509 & 173.047 & 3,71 \\
\hline & & & & Kairuán & 72.254 & 117.903 & 2,48 \\
\hline
\end{tabular}

Fuente: Argelia: ONS, Office National des Statistiques: statistiques sociales, Recensement Général de la Population, 2008 http://www.ons.dz/. Marruecos: Haut-Commissariat au Plan, Recensement Général de la Population et de I'Habitat, 2004. http://www.hcp.ma/. Túnez: Institut National de la Statistique, Recensement de la Population, 2004. http://www.ins.nat.tn/. Elaboración propia. 
Atendiendo al Índice de Primacía ${ }^{17}$ o dominio de la ciudad principal sobre el resto de asentamientos, se advierte una tendencia a la macrocefalia en Marruecos (63,95\%); ya que por su por dimensión, por su conectividad internacional y por su situación geográfica en el Atlántico destaca su gran área metropolitana Rabat-Casablanca o Área Metropolitana Atlántica Marroquí. Ocupa las densas regiones de Rabat-Salé-Zemmour-Zaer y de Gran Casablanca y concentra aproximadamente a más de seis millones y medio de habitantes, es decir, el 40\% de la población urbana del país. El peso de esta aglomeración se refleja en ser la principal receptora de inversiones extranjeras del país, tanto por la deslocalización de empresas extranjeras del sector automovilístico o de telecomunicaciones ${ }^{18}$, como por construcción de infraestructuras, muestra de ello es la existencia de dos aeropuertos internacionales en menos de 120 kilómetros o la consolidación de un corredor ferroviario y de carreteras que articula a la metrópolis y que junto a su red de puertos, domina económicamente el país. Dicha descripción no oculta las enormes disparidades del sistema de asentamientos y sus posibles repercusiones en cuanto a la accesibilidad y la prestación de servicios de la población en otras partes de Marruecos, especialmente en las de mayor tradición emigrante y/o situadas al norte del país. "Cette accumulation d'hommes, d'espaces bâtis, d'équipements, d'activités, cette convergente majeure de flux en provenance de tout le pays pourraient être source d'un violent déséquilibre territorial et fonctionnel, (Troin, et al., 2006: 294).

Por su parte, el Índice de Primacía para Túnez (56,71\%) y Argelia (59,73\%) no alcanza los niveles macrocefálicos de Marruecos y la tendencia es a una red urbana más repartida entre las provincias litorales y pre-litorales. Túnez es el país del Magreb Histórico que menor incremento de población urbana ha registrado en los últimos años, el 4,89\% pese a ser el más urbanizado a comienzos de siglo (tabla 1). No obstante, dicho incremento ejemplifica la consolidación de su carácter urbano y no agrario. Según Troin y otros (2006: 283) de todas las capitales magrebíes, la ciudad de Túnez es la que cuenta con mayor número de actividades económicas y poder de decisión, hecho que se explica por el fuerte centralismo de época colonial y su continuación tras la independencia. Pero sin duda, y al igual que en Argelia, el clima y la situación geográfica de las ciudades meridionales y del interior, explican las bajas densidades demográfi-

17. El Índice de Primacía mide el dominio de la ciudad más poblada de un sistema urbano sobre el resto de asentamientos de ese mismo sistema. El Índice de Primacía se expresa:

$$
I p=\frac{P_{1}}{4 \sum_{i=1} P i} x 100
$$

Donde Ip = es el Índice de Primacía, P1 = la población de la ciudad con más habitantes del sistema urbano $4 \Sigma \mathrm{i}={ }_{4} \mathrm{Pi}=$ la suma de los cuatro asentamientos más poblados incluido el principal. Los valores que tienden a 100 suponen una tendencia a la macrocefalia, mientras que los que lo hacen hacia 25 muestran una mayor tendencia a la policefalia.

18. De la cantidad de artículos de prensa que informan y opinan sobre la deslocalización empresarial en Marruecos se puede destacar, entre otras, la noticia del diario LE PARISIEN fechada el 11/03/2010: Le Maroc, royaume des délocalisations françaises. 
cas y la primacía del litoral frente al interior. Esta dualidad, sin embargo, no es completamente aplicable al caso argelino, en donde la preponderancia de la capital es menos decisiva que la de Casablanca-Rabat o la de Túnez (Semmoud, 2010: 253), ya que en ese sentido Argelia junto con Turquía, es el país de mayoría musulmana del Mediterráneo donde el dominio litoral es menos importante (Semmoud: 2010: 241). En efecto, Argelia, el décimo país del mundo por extensión $\left(2.381 .741 \mathrm{~km}^{2}\right)$ y el primero de África y del mundo árabe, es complejo desde múltiples puntos de vista; y a ese respecto su poblamiento y red de ciudades están influidos por múltiples condicionantes de tipo histórico, geográfico, cultural, político o económico. Al margen de Argel y Orán, separadas por 350 kilómetros, ninguna del resto de sus ciudades superan los 750.000 habitantes, hecho que se produce en el país del Magreb Central que más intensamente ha aumentado su tasa de población urbana desde comienzos del siglo XXI. En relación a esta dinámica, la capital argelina es la que mayor incremento demográfico ha registrado en los últimos años de las capitales magrebíes, un 1,26\% anual entre 1998 y 2008, lo que se relaciona con las graves disfunciones que Troin y otros (2006: 274) identifican para Argel, pero que bien podrían aplicarse a otros territorios del Mediterráneo: 1. Una crisis del hábitat que se traduce en dificultades de acceso a una vivienda digna, 2. Una crisis de los transportes que afecta a la movilidad de los ciudadanos y de las mercancías y 3. Una crisis ecológica visible en la contaminación atmosférica y en la captación y evacuación del agua de abastecimiento urbano; en definitiva, disfunciones que podrían paliarse mediante una políticas de ordenación del territorio distintas a las que han sido más habituales y que subyacen bajo las demandas ciudadanas de los últimos años.

\section{Conclusiones}

Pese a las múltiples y profundas diferencias y similitudes que existen entre los países y territorios bañados por el Mediterráneo, es posible aceptar que la convergencia de las transiciones demográficas es un hecho que, con distintas velocidades, tiende a unificar el devenir sociodemográfico de la región. Además, es incuestionable que para hacer frente a los desafíos a los que en particular se enfrentan los países de mayoría musulmana del Magreb y de Oriente Próximo, y que fueron anunciados en 2009 por el Informe sobre Desarrollo Humano Árabe de la ONU, es necesario tener en cuenta cuál ha sido la evolución reciente de las estructuras y dinámicas demográficas en el territorio; pues no en vano, son las poblaciones las que en definitiva deben alcanzar las mayores cotas de desarrollo.

Desde mediados del siglo XX hasta comienzos de la segunda década del siglo XXI el peso relativo de la población de los países mediterráneos respecto del total mundial ha disminuido a favor de otras áreas geográficas mundiales. Esa pérdida de peso demográfico, que no geopolítico, ha sido resultado de importantes procesos de reducción de la fecundidad y de aumento de la esperanza de vida; traduciéndose, a pe- 
sar de significativas diferencias nacionales, en unas estructuras demográficas envejecidas en el caso de los países europeos y en una estructura demográfica compuesta por importantes grupos de población adulta-joven que constituyen un dividendo demográfico muy abultado de las poblaciones potencialmente activas de los países del sur y del este Mediterráneo. En ese sentido, los saldos migratorios que se observan a ambas orillas del mar no han sido capaces de resolver los problemas geodemográficos de los países europeos ni tampoco lograr una contención social en los países musulmanes como demuestran el malestar latente y las revueltas recientes en algunas ciudades por parte de una población, generalmente más joven, más formada, más informada y con mayores expectativas laborales.

Centrándonos en los países del Magreb Central cabe destacar la importante correlación que se observa entre el incremento del Índice de Desarrollo Humano y la reducción en la fecundidad de las mujeres y, en menor medida, de las diferencias de género. En efecto, y a las puertas de una segunda transición demográfica, las proyecciones de población a corto plazo que realizan los organismos internacionales, preveen una mayor presencia, al menos demográfica, de las mujeres. Las cuales, y en un contexto de lento pero claro proceso de secularización, adquieren mayor protagonismo en unas sociedades cada vez más urbanizadas.

Finalmente, cabe señalar que desde el punto de vista territorial, y teniendo en cuenta las limitaciones de las fuentes estadísticas utilizadas en este trabajo, la urbanización reciente en los países del Magreb histórico presenta los típicos problemas sociales y ambientales asociados, por un lado, a la dispersión metropolitana y, por otro lado, a la concentración de funciones en las ciudades principales. Pero al margen de esta tendencia conviene resaltar que en la actualidad y pese a la primacía que ejercen las capitales y/o las grandes aglomeraciones urbanas litorales sobre sus respectivos territorios nacionales, se advierte la emergencia de otras regiones cuyas ciudades principales recogen el testigo de los mayores incrementos de población urbana en un contexto de modernización social y demográfica en donde la feminización de la sociedad y la existencia de importantes dividendos de población potencialmente activa son claves para entender y gestionar el presente de la región.

\section{Bibliografía}

Álvarez-Ossorio, I. (2011) El mito de la conflictividad del mundo árabe: de la época colonial a las revueltas populares. Investigaciones Geográficas, n. 55, pp. 55-70.

Bardot, C.; Crouzet, G.; Perrier, F. (2010) Moyen-Orient \& Maghreb. Paris, Pearson, 176 p.

Bennafla, K.; Pagès-el Karoui, D.; Sanmartin, O. (2011) Géopolitique du Maghreb et du MoyenOrient. Paris, Armand Colin, 230 p.

Braudel, F. (1987) El Mediterráneo y el mundo mediterráneo en la época de Felipe II. México D.F. Fondo de Cultura Económica, 858 p. (1 $1^{\underline{a}}$ Ed. en francés 1949. $1^{\underline{a}}$ Ed. en español 1953). 
Courbage, Y.; Todd, E. (2009) Encuentro de civilizaciones. Madrid, FOCA, 175 p.

Courbage, Y. (2009) "Nuevas reflexiones sobre los escenarios demográficos de la orilla sur del Mediterráneo: certezas e incertidumbres", en Jiménez, T. (Ed.): Población y desarrollo en el Mediterráneo. Transiciones demográficas y desigualdades socioeconómicas. Barcelona, Icaria, pp. 71-95.

Domingo, A. (2011) El futuro demográfico del Mediterráneo. Una mirada periférica desde la experiencia de la crisis. IECA. Información Estadística y Cartográfica de Andalucía, n. 1, pp. 102-104.

Dumont, G.-F.; Montenay, Y. (2011) Le Maghreb, une géopolitique éclatée. Geostratégiques, n. 32 (3), pp. 29-65.

Fargues, P. (2008): Emerging demographic patterns across the Mediterranean and their implications for migration through 2030. Washington DC, Migration Policy Institute. Disponible en http://www.migrationpolicy.org/pubs/fargues-paper.pdf [consulta: 15 de mayo].

Fargues, P. (2009) "Migraciones en el Mediterráneo: un panorama general", en Jiménez, T. (Ed.): Población y desarrollo en el Mediterráneo. Transiciones demográficas y desigualdades socioeconómicas. Barcelona, Icaria, pp. 267-283.

George, P. (2007) Diccionario Akal de Geografía. Madrid, Akal, 622 p. (1ํㅡㄹ Ed. en francés 1970. $1^{\underline{a}}$ Ed. en español 1991).

Gil, F.; Domingo, A.; Bayona, J. (2011) Flujos migratorios en el Mediterráneo Occidental: ¿causas demográficas, sociales o económicas? Cuadernos Geográficos, n. 48, pp. 47-67.

Griffin, K. (2001) Desarrollo humano: origen, evolución e impacto. En Unceta, K.; Ibarra, P. (Coords.): Ensayos sobre el desarrollo bumano. Barcelona, Icaria, pp. 25-40.

Huntington, S. (2007) El choque de civilizaciones y la reconfiguración del orden mundial. Barcelona, Paidos, 487 p. (1 ${ }^{\text {a }}$ Ed. 1997)

Kamel Al-Sayyid, M. (2009) Población, desarrollo humano y seguridad humana en los países árabes del Mediterráneo. En Jiménez, T. (Ed.): Población y desarrollo en el Mediterráneo. Transiciones demográficas y desigualdades socioeconómicas. Barcelona, Icaria, pp. 21-26.

Mansevelt-Beck, J.; De Mas, P. (2001) The Strait of Gibraltar: Europe's Río Grande. En King, R.; De Mas, P.; Mansvelt-Beck, J.: Geography, environment and development in the Mediterranean. Brighton, Sussex Academic Press, pp. 117-135

Lacoste, Y. (2010) El Mediterráneo, una cuenca con identidad propia. En Greilsamer, L.; Denis, J.-P. (Ed.) El atlas de las civilizaciones. Valencia, Fundación Mondiplo, pp. 100-101.

Lacoste, Y.; Lacoste, C. (1991) L'Etat du Maghreb. Paris, La Découverte, 572 p.

Locoh, T.; Ouadah, Z. (2010) Familia y relaciones de género en el Magreb. En Bessis, S.; Martín, G. (Coords.) Mujer y familia en las sociedades árabes actuales. Barcelona, Edicions Bellaterra, pp. 13-44.

López Ontiveros, A. (2008) Reflexiones y discursos sobre el mediterráneo desde la Península Ibérica y Andalucía. En VV.AA.: España y el Mediterráneo: aportación española al XXXI congreso de la UGI. Madrid, Ministerio de Fomento, Instituto Geográfico Nacional, pp. 93-97. 
Méndez, R. (2011) Tensiones y conflictos armados en el sistema mundial: una perspectiva geopolítica. Investigaciones Geográficas, n. 55, pp. 19-37.

Mutin, G. (2009) Géopolitique du Monde arabe. Paris, Ellipses, 245 p.

Ouadah-Bedidi, Z. ; Vallin, J. ; Bouchoucha, I. (2012) La fécondité au Maghreb: nouvelle surprise. Population \& Sociétés, n. 486. Disponible en http://www.ined.fr/fichier/t_publication/1581/publi_pdf1_486.pdf [consulta: 15 de mayo 2013].

Pool, I. (2007) Demographic dividends: determinants of development or merely windows of opportunity? Aeging Horizons, n. 7, pp. 28-35

Reig-Martínez, E. (2013) Social and Economic Wellbeing in Europe and the Mediterranean Basin: Building an Enlarged Human Development Indicator. Social Indicators Research, n. 111 (2), pp. 527-547.

Roy, O. (2009) Islam: la revolución invisible. En Denis, J.-P.; Frachon, A. (Ed.) El Atlas de las religiones. Buenos Aires, Capital Intelectual, pp. 58-59.

Semmoud, B. (2010) Maghreb et Moyen-Orient dans la mondialisation. Paris, Armand Colin, 318 p.

Troin, J.-F.; Bisson, J.; Brüle, J.-C.; Escallier, R.; Fontaine, J.; Signoles, P. (2006) Le Grand Maghreb. Paris, Armand Colin, 384 p.

UNITED NATIONS DEVELOPMENT PROGRAMME (2009) Arab Human Development Report, 2009: Challenges to Human Security in the Arab Countries. New York, United Nations Publications, p. 265. Disponible en http://www.arab-hdr.org/contents/ [consulta: 15 de mayo]. 